\title{
AN ASCA OBSERVATION OF THE ECLIPSING DWARF NOVA HT CAS
}

\author{
K. MUKAI ${ }^{1}$, E. M. SCHLEGEL ${ }^{1,2}$, J. H. SWANK ${ }^{1}$, T. NAYLOR $^{3}$, \\ JANET H. WOOD ${ }^{3}$ \\ 1. Laboratory for High Energy Astrophysics, NASA \\ Goddard Space Flight Center, Greenbelt, MD 20771, USA \\ 2. Also Universities Space Research Association \\ 3. Department of Physics, University of Keele, \\ Keele, Staffordshire, ST5 5BG, UK
}

\begin{abstract}
We report on a 1-day ASCA observation of the eclipsing dwarf nova HT Cas. We confirm the presence of an X-ray eclipse, which is narrow and deep. The data are consistent with the $\mathrm{X}$-rays originating entirely from the immediate neighborhood of the white dwarf. We draw some preliminary conclusions on the boundary layer and other relevant issues.
\end{abstract}

\section{Introduction}

Hard X-ray emission in quiescent dwarf novae (e.g. van Teeseling 1996) is most often interpreted as arising from the optically thin boundary layer between the accretion disk and the white dwarf. However, there has been little direct observational evidence for this interpretation. Moreover, the disk instability theory of dwarf nova outbursts predicts a mass accretion rate onto the white dwarf that is much lower than is required by $\mathrm{X}$-ray observations. Instead, coronal siphon flow models suggest that the boundary layer in the classical sense may not exist (Meyer \& Meyer-Hofmeister 1994). There is thus a strong motivation for further observational studies of the hard X-rays from quiescent dwarf novae, particularly eclipsing systems which provide a clean experiment.

We have chosen HT Cas as our target, because it is probably the brightest system in hard $\mathrm{X}$-rays among well-studied, deeply eclipsing dwarf novae (i.e. systems in which the white dwarf is eclipsed, not just parts of the accretion disk). In a previous study, Wood et al. (1995) observed HT Cas with ROSAT and revealed the presence of an X-ray eclipse; however, HT Cas was in an unusual low state at the time. This led to lower-than-expected

A. Evans and J. H. Wood (eds.), Cataclysmic Variables and Related Objects, 269-272. (C) 1996 Kluwer Academic Publishers. Printed in the Netherlands. 
data quality; also a doubt remained about the relevance of the data to the normal state. To follow up, we utilized the ASCA satellite (see e.g. Ishida 1996) which has imaging (i.e. low background) capabilities up to $\sim 10 \mathrm{keV}$, with roughly 4 times higher effective area than ROSAT.

\section{Observation}

HT Cas was observed with ASCA between 1994 September 6 17:25 UT and 1994 September 7 17:10 UT. The observation was planned to maximize eclipse coverage: 6 eclipses were covered completely as well as parts of 2 more. Standard screening criteria have been applied to produce $31 \mathrm{ks}$ of good SIS data and 34 ks of good GIS data. Light curves and spectra were then extracted from circular regions with $\sim 3.5$ and $\sim 6.0$ arcmin radii respectively, for SIS and GIS. Background subtraction is a major issue for fainter sources: blank sky and local background subtraction methods have been tried to give an indication of systematics.

In addition, optical CCD photometry was performed several times during the August... October period at Keele Observatory. HT Cas was found to be in normal quiescence throughout this period ( $V \sim 16.5 \mathrm{mag}$ out of eclipse). For phasing, we apply a correction of $\sim 30 \mathrm{~s}$ to the ephemeris of Horne, Wood \& Stiening (1991), as derived from recent optical photometry (Wood et al. 1995).

\section{Results}

The folded ASCA light curve of HT Cas is shown in Fig. 1. We discuss the out-of-eclipse behavior first, then discuss the eclipse.

\subsection{THE OUT-OF-ECLIPSE BEHAVIOR}

The orbital phase interval $0.2 \ldots 0.4$ was covered rather poorly, hence the light curve is noisier during this time. Taking this into consideration, we have found no evidence for a systematic variability outside the eclipse in folded and unfolded light curves. We have also examined light curves for soft $(E<2 \mathrm{keV})$ and hard $(E>2 \mathrm{keV})$ bands, and found no systematic variability in hardness ratio.

We have therefore extracted the average out-of-eclipse spectra from the 4 instruments, and fitted a variety of standard models. The best fit temperature with the Raymond-Smith model is $10.5_{-1.4}^{+1.5} \mathrm{keV}$ with local background subtraction, or $8.7_{-1.0}^{+1.1} \mathrm{keV}$ with blank sky background, i.e. HT Cas had a hard spectrum for a non-magnetic CV at the time of the ASCA observation. At such temperatures, plasma models predict a spectrum dominated by the bremsstrahlung continuum, with $6.7 \mathrm{keV}$ (He-like) and $6.97 \mathrm{keV}$ 


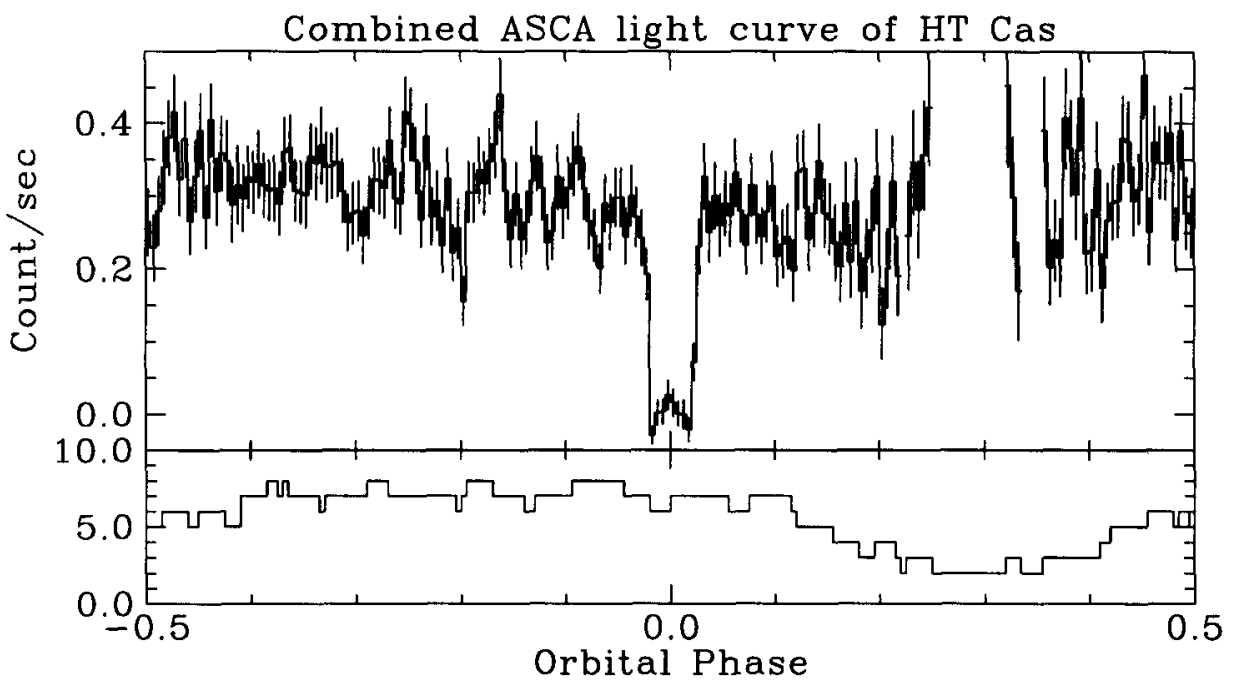

Figure 1. Folded X-ray light curve of HT Cas: data from all 4 ASCA instruments have been combined, folded on the ephemeris of Horne et al. with $30 \mathrm{~s}$ correction derived from recent optical photometry. The lower panel shows the number of different cycles that were combined to derive the value for that bin. There are 200 phase bins of $\sim 32 \mathrm{~s}$ per bin.

(H-like) lines of $\mathrm{Fe} \mathrm{K}$, which is consistent with the bremsstrahlung fit we obtained; the lines were approximated by a single, broad Gaussian as the statistical quality of the data was not good enough for a multi-component fit. We therefore conclude that the X-ray emission from HT Cas was dominated by $\sim 10 \mathrm{keV}$ plasma. Our attempt to add a second, lower-temperature component did not result in an improved fit.

The source is absorbed with $N_{\mathrm{H}}=3.3 \pm 0.310^{21} \mathrm{~cm}^{-2}$, independent of whether bremsstrahlung or the Raymond-Smith model was used. The measured flux (average of out-of-eclipse data) is $3.510^{-12} \mathrm{ergs} \mathrm{cm}^{-2} \mathrm{~s}^{-1}$ in the $2-10 \mathrm{keV}$ range, corresponding to an inferred bolometric flux of $1.0710^{-11}$ ergs $\mathrm{cm}^{-2} \mathrm{~s}^{-1}$. Adopting a distance of $165 \mathrm{pc}$, the hard X-ray luminosity of $\mathrm{HT}$ Cas was $\sim 3.5410^{31} \mathrm{ergs} \mathrm{s}^{-1}$; if this was half the accretion luminosity onto a $0.64 \mathrm{M}_{\odot}$ (Horne et al. 1991) white dwarf, then an accretion rate was $7.110^{14} \mathrm{~g} \mathrm{~s}^{-1}$ or $1.110^{-11} \mathrm{M}_{\odot} \mathrm{yr}^{-1}$.

\subsection{THE ECLIPSE}

The eclipse appears total and narrow (Fig. 1). Here we report preliminary results from our ongoing effort to quantify this statement.

First, how total is the eclipse? The errors are dominated by the uncertainties in the true background level. We have therefore measured the 
mid-eclipse flux in two types of instruments independently, using two background subtraction methods, and two energy bands. With the SIS, we obtain a more tightly constrained result: $-2.2 \pm 5.3 \%$ of the out-of-eclipse average in the $0.7 \ldots 5 \mathrm{keV}$ band (which was selected by eye to maximize signal-to-noise) using local or blank sky background. The values for GIS were $3.8 \pm 10.1 \%$ and $1.8 \pm 10.3 \%$, respectively, using local and blank sky backgrounds. Thus the eclipse is consistent with being total, although a very low residual flux (up to $\sim 3 \%$ ) cannot be excluded.

A piece-wise linear fit to the light curve indicates the eclipse duration (mid-ingress to mid-egress) of 0.045 cycle, as compared with 0.0489 cycle for the optical eclipse of the white dwarf photosphere (Horne et al. 1991). As a first approximation, a spherical emission region with a radius $R_{\mathrm{wd}}$ (as determined in the optical), using the system geometry derived in the optical, can reproduce the parameters of the X-ray eclipse. Thus, we conclude that the X-ray emission is localized to the white dwarf and has a size comparable to it. However, there are indications that the X-ray eclipse is perhaps too narrow: our continued investigation will hopefully resolve this mystery.

\section{Discussion}

The ASCA spectrum of HT Cas indicates an absorption much greater than is seen in the ROSAT spectrum. It indicates that, at the time of the ASCA observation, there was an absorbing cloud within the binary system. Given the high inclination of HT Cas, it is reasonable to suppose that an increased accretion rate is accompanied by a thickening of the disk or the boundary layer, resulting in the observed increase in absorption.

ROSAT and ASCA observations have established that much of the hard $\mathrm{X}$-ray flux in HT Cas in quiescence originates very near the white dwarf, in normal quiescence and in a low state. The ASCA measurement of the flux indicates that the accretion rate onto the white dwarf must be relatively high. The boundary layer model of hard X-ray emission is a natural interpretation for the X-ray data, although maintaining this level of accretion may cause problems for the disk instability model of dwarf nova outbursts. The coronal siphon model will be consistent with the data only if the extended accretion disk corona that is an essential ingredient of the model is shown not to emit a significant fraction of the X-rays.

\section{References}

Horne, K., Wood, J.H., Steining, R.F., 1991, Ap. J., 378, 271

Ishida, M., 1996, these proceedings, p259

Meyer, F., Meyer-Hofmeister, E., 1994, A\&A, 288, 175

van Teeseling, A., 1996, these proceedings, p273

Wood, J.H., Naylor, T., Hassall, B.J.M., Ramseyer, T.F., 1995, MNRAS, 273, 772 